\title{
The Proximal Tibia Loses Bone Mineral Density After Anterior Cruciate Ligament Injury: Measurement Technique and Validation of a Quantitative Computed Tomography Method
}

\author{
Erick M. Marigi, M.D., David R. Holmes, Ph.D., Naveen Murthy, M.D., Bruce A. Levy, M.D., \\ Michael J. Stuart, M.D., Diane L. Dahm, M.D., Peter C. Rhee, D.O., M.Sc., and \\ Aaron J. Krych, M.D.
}

Purpose: To develop a standardized method for tibial tunnel volumetric bone mineral density (BMD) analysis with
quantitative computed tomography (qCT) using cadaveric specimens to provide validation of this technique on a healthy
control population and to determine whether osteopenia occurs following an anterior cruciate ligament (ACL) injur-
Y. Methods: qCT was used to develop a volumetric BMD $\left(\mathrm{mg} / \mathrm{cm}^{3}\right.$ ) measurement technique throughout the region of a
standard tibial tunnel. This method was applied to $90 \mathrm{lower}$ extremities, including 10 matched cadaveric knees, 10
matched healthy knees, $25 \mathrm{ACL}$-injured knees, and $25 \mathrm{contralateral} \mathrm{ACL-uninjured} \mathrm{knees.} \mathrm{The} \mathrm{mean} \mathrm{total} \mathrm{and} \mathrm{segmental}$
(proximal, middle, and distal) tibial tunnel BMD were analyzed. Results: The mean entire tibial tunnel BMD measured
$165.8 \pm 30.5 \mathrm{mg} / \mathrm{cm}^{3}$ (cadaver), $255.9 \pm 28.2 \mathrm{mg} / \mathrm{cm}^{3}$ (healthy control), $290.3 \pm 36.4 \mathrm{mg} / \mathrm{cm}^{3}$ (ACL-injured), and 300.1
\pm 35.1 (ACL-uninjured). Segmental tibial tunnel BMD demonstrated distal one-third segments as the greatest areas of
BMD, followed by proximal one-third, and middle one-third for all cohorts with all pairwise comparisons $(P<.001)$. The
mean BMD was significantly greater in the uninjured extremity compared with the injured extremity in the entire tunnel
(290.3 vs $300.1 ; P<.001$ ), proximal $(271.2$ vs $279.1 ; P=.002)$, middle $(167.6$ vs $179.6 ; P<.001$ ), and distal segments
(432.7 vs $441.7 ; P=.004$ ) at an average of 8 weeks following ACL injury. Conclusions: A standardized method to
quantitatively measure the volumetric BMD within the region of a standard tibial tunnel for ACL reconstruction was

From the Departments of Orthopedic Surgery (E.M.M., B.A.L., M.J.S., D.L.D., P.C.R., A.J.K.), Biomedical Imaging Resource (D.R.H.), and Radiology (N.M.), Mayo Clinic, Rochester, Minnesota, U.S.A.

The authors report the following potential conflicts of interest or sources of funding: E.M.M. reports nonfinancial support from Orthofix Medical, outside the submitted work. B.A.L reports personal fees from Arthrex, IP royalties, paid consultant, and grants from Biomet: research support, other from Clinical Orthopaedics and Related Research: editorial or governing board, other from Journal of Knee Surgery; editorial or governing board, other from Knee Surgery, Sports Traumatology, Arthroscopy; editorial or governing board, other from Orthopedics Today; editorial or governing board, grants, and personal fees from Smith $\theta$ Nephew; paid consultant, research support, and grants from Stryker; research support and personal fees from Linvatec; and faculty/speaker and personal fees from COVR Medical LLC, outside the submitted work. M.J.S. reports other from Arthrex, during the conduct of the study; other from American Journal of Sports Medicine, grants and personal fees from Arthrex, and grants from Stryker, outside the submitted work. P.C.R. reports nonfinancial support from American Association for Hand Surgery, nonfinancial support from American Society for Surgery of the Hand, nonfinancial support from Clinical Orthopaedic Society, and personal fees from Trimed, outside the submitted work. D.L.D. reports other from the AJSM Medical Publishing Board of Trustees, from American Orthopaedic Society for Sports Medicine, grants from Arthrex, other from the NBA/GE Strategic Advisory Board, personal fees from Tenex Health, personal fees from Sonex Health, LLC, and nonfinancial support from GE Healthcare, outside the submitted work. A.J.K. reports other from Arthrex, during the conduct of the study; grants from Aesculap/B. Braun; other from American Journal of Sports Medicine; personal fees and other from Arthrex; grants from the Arthritis Foundation; grants from Ceterix; grants from Histogenics; other from International Cartilage Repair Society, International Society of Arthroscopy, Knee Surgery, and Orthopaedic Sports Medicine, and Minnesota Orthopedic Society; personal fees and other from the Musculoskeletal Transplant Foundation; personal fees from Vericel, DePuy, and JRF; grants from Exactech and Gemini Medical, and personal fees from Responsive Arthroscopy and Joint Restoration Foundation, outside the submitted work. The authors would like to acknowledge the support from the National Institutes of Health (NIH) (R01AR055563). Its contents are solely the responsibility of the authors and do not necessarily represent the official views of the NIH. Full ICMJE author disclosure forms are available for this article online, as supplementary material.

Primary Location where this investigation was performed: Mayo Clinic, Rochester, Minnesota, U.S.A.

Received June 19, 2021; accepted September 20, 2021.

Address correspondence to Aaron J. Krych, M.D., 200 First St. SW, Rochester, MN 55905.E-mail:Krych.aaron@mayo.edu

(C) 2021 THE AUTHORS. Published by Elsevier Inc. on behalf of the Arthroscopy Association of North America. This is an open access article under the CC BY-NC-ND license (http://creativecommons.org/licenses/by-nc-nd/4.0/). 2666-061X/21879

https://doi.org/10.1016/j.asmr.2021.09.010 
successfully developed and validated. Significant osteopenia of the injured knee occurs following ACL injury when compared with the contralateral uninjured knee. This observation has potential clinical implications for ACL graft tibial fixation and healing. Level of Evidence: Descriptive diagnostic study, Level III.

A nterior cruciate ligament (ACL) disruption is a devastating injury that results in substantial bone mineral density (BMD) loss about the knee that can persist after ACL reconstruction. ${ }^{1-4}$ The etiology has been attributed to catabolic stress hormones from the inciting injury, subsequent surgery, immobilization, and altered injured extremity weight-bearing. ${ }^{4-7}$ Multiple studies have evaluated BMD changes around the knee after an ACL injury and/or reconstruction and have observed the greatest bone loss in the proximal tibia. $^{2-4,8}$ Likewise, biomechanical studies have also confirmed that the proximal tibia is the weak link for graft fixation in ACL reconstruction. ${ }^{9-11}$ Clinically, decreases in BMD may also play a role in the propensity for postoperative tibial fractures and decreased strength of interference screw fixation. ${ }^{12,13}$ As a result, certain institutions have begun to use BMD measurements as a decision-making guide for graft fixation. ${ }^{13}$

Biomechanical investigations of tibial fixation constructs for ACL reconstruction have been performed demonstrating a lower mean load to failure in cadaveric tibias with a BMD less than $600 \mathrm{mg} / \mathrm{cm}^{2}(201 \mathrm{~N})$ and in older cadaveric specimens after ACL reconstruction using a bone-patellar tendon-bone graft. ${ }^{9,14,15}$ Despite this, biomechanical analysis of tibial fixation constructs warrants an accurate assessment of BMD within the region of graft fixation. Dual energy x-ray absorptiometry (DEXA) has been widely employed to evaluate the proximal tibial $\mathrm{BMD}$ of test specimens before biomechanical testing of ACL reconstruction fixation constructs, but this method is highly inaccurate. The planar nature of BMD assessment limits the ability of DEXA to differentiate cortical from cancellous bone, which is of critical importance as interference screw fixation strength improves with purchase into dense cortical or subchondral bone. ${ }^{16,17}$ In addition, BMD reports with DEXA are based upon algorithms intended for analysis of the lumbar spine or distal radius, not the proximal tibia. ${ }^{18}$ As such, most biomechanical and clinical studies involving ACL injury and reconstruction have depended upon this imprecise modality for BMD assessment within the proximal tibia. ${ }^{1-4,6,8-10,19,20}$

Quantitative computed tomography (qCT) has emerged as a precise and valid method to measure BMD within the peripheral long bones, with the ability to distinguish cortical from cancellous bone, and has been used in many biomechanical and clinical ACLreconstruction studies to overcome the limitations of DEXA. ${ }^{21-29}$ However, no standardized method of in vitro or in vivo volumetric BMD assessment have been described that accurately measures the region within the entire tibial tunnel. Furthermore, baseline values for cadaveric specimens, a healthy uninjured patient population, or an ACL-injured patient population have yet to be defined. The purposes of this study were to develop a standardized method for tibial tunnel volumetric $\mathrm{BMD}$ analysis with $\mathrm{qCT}$ using cadaveric specimens, to provide validation of this technique on a healthy control population, and to determine whether osteopenia occurs following an ACL injury. We hypothesized that qCT would be used to assess volumetric BMD within the region of a standard tibial tunnel for ACL reconstruction and detect osteopenia following an ACL injury.

\section{Methods}

\section{Study Population}

\section{Cadaveric Specimens}

Twenty fresh-frozen cadaveric lower extremities (10 matched pairs, 5 male, 5 female) with a mean age of $76 \pm 8.4$ years (range: 60-85 years), were obtained from our institutional cadaver bank. The specimens were grossly inspected by an orthopaedic surgeon (P.C.R) and selected only if there was no physical evidence or medical documentation of a previous knee surgery. The soft tissues (skin, subcutaneous fat, muscle, menisci, collateral and cruciate ligaments) adjacent to the proximal tibia were left intact to simulate an environment similar to in vivo qCT scanning.

\section{Validation in a Healthy Control Subject Group}

After approval by the institutional and radiation safety review board (Mayo \#08-008067), 10 healthy adult volunteers ( 7 male, 3 female) with a mean age of $35.1 \pm 10.2$ years (range: $24-55$ years) were recruited and underwent qCT scanning of the bilateral knees ( $\mathrm{n}=20$ knees). Volunteers were excluded if there was a positive history of previous knee surgery or significant injury requiring immobilization in either lower extremities, use of any medication known to affect BMD, symptomatic or known lumbar or hip pathology, history of rheumatologic or inflammatory disease, or if the patient was pregnant. Each patient completed a questionnaire evaluating their knee function (Lysholm knee score and the International Knee Documentation Committee [IKDC] subjective knee evaluation score) $)^{30,31}$ and activity level (Tegner and University of California - Los Angeles activity level scores [UCLA]). ${ }^{32}$ Epidemiologic data (body mass index 

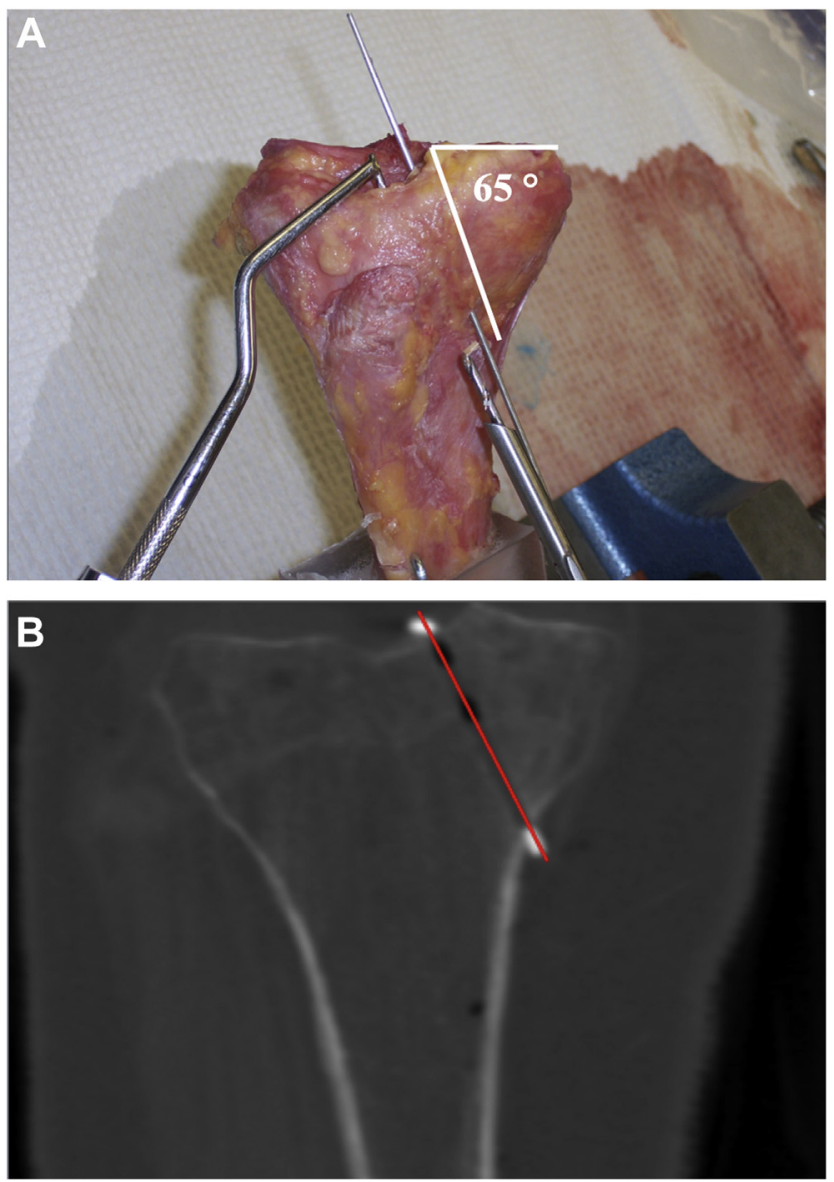

Fig 1. Demonstration of cadaveric tibial tunnel identification. (A) A 2.4-mm guide pin is directed towards the ACL footprint at $55^{\circ}$ from the articular surface in the sagittal plane and $65^{\circ}$ (white line) from the medial tibial plateau in the coronal plane. Soft tissues have been removed for illustrative purposes. (B) Radio-opaque markers are placed at the proximal and distal ends of the guide pin tract (red line) after pin removal. (ACL, anterior cruciate ligament.)

[BMI]) and age) was obtained to serve as normative data for a healthy (uninjured) population.

\section{ACL Study Group}

Concurrently, 25 patients (20 male and 5 female) with a mean age of $25.9 \pm 7.7$ years (range: 18-48 years) who sustained a complete tear of the ACL were enrolled into the ACL-injured patient study group by one of the 3 senior authors (D.L.D., B.A.L., or M.J.S.) at the time of initial orthopaedic sports medicine consultation over a 3-year period. qCT scans were obtained of the uninjured $(n=25)$ and injured extremity $(n=25)$ before ACL reconstructive surgery. Inclusion criteria consisted of patient age $\geq 18$ years old, unilateral knee injury, and magnetic resonance imaging (MRI) confirmation of a complete (grade III) ACL rupture. Patients were excluded if there was MRI evidence of a concomitant rupture (grade III) of the remaining cruciate or collateral ligaments, history of previous knee surgery or significant injury requiring immobilization in either lower extremities, use of any medication known to affect BMD, symptomatic or known lumbar or hip pathology, history of rheumatologic or inflammatory disease, or if the patient was pregnant. All patients completed postinjury knee function scores (Lysholm and IKDC) as well as pre- and postinjury activity level (Tegner and UCLA) scores. Other epidemiologic factors (age and BMI) were obtained to serve as normative data for an ACL disrupted patient population.

\section{Cadaveric Tibial Tunnel BMD Assessment Technique}

A single surgeon performed all the procedures. The tibial tunnel was identified and marked with a $2.4-\mathrm{mm}$ guide pin drilled into the proximal tibia using a tibial tunnel guide (Arthrex, Naples, FL) set at $55^{\circ}$. The pin was inserted at $65^{\circ}$ from the medial tibial plateau in the coronal plane (Fig 1A). ${ }^{33}$ The pin was directed toward the remnant of the ACL insertion on the tibial footprint. The pin was removed and radio-opaque markers were placed at the proximal and distal ends of the guide pin tract (Fig 1B). ${ }^{34,35}$

A single postimaging analysis technician performed the image processing and Hounsfield unit calculation with repeat processing and analysis at a minimum of 1 week from the initial tests. Intraclass correlation coefficients were then calculated based on sampling of baseline and repeat measurements with calculated intraobserved intraclass correlation coefficients demonstrating strong correlations, with values greater than 0.8. qCT was used to assess BMD within the region of the previously identified tibial tunnel. ${ }^{36}$ The qCT scans were acquired with a CT scanner protocol (Somatom Definition SD; Seimens Healthcare, Forcheim, Germany) set at collimation of $64 \times 0.6$, pitch of $1 \mathrm{~mm}$, kernel of B70s, and reconstruction increment of $0.5 \mathrm{~mm}$. The cadaveric tibias were placed on top of a 6level hydroxyapatite phantom (Model 3 CT Phantom; Mindways Software, Inc., San Francisco, CA) and were visualized within the same field of view. ${ }^{37}$

The region of interest (RoI) was a 3-mm thick ring between a $9 \mathrm{~mm}$ and $15 \mathrm{~mm}$ circle per axial slice centered over the guide pin tract (Fig 2A). Custom software was used to stack the ring RoIs in series along the trajectory of the guide pin tract to provide a cylindrical RoI within the peripheral bone of a potential 9-mm tibial tunnel, at the site of graft-bone or screw-bone interface. The cylindrical RoI was divided into 3 equal regions (proximal one-third, middle one-third, and distal one-third) for segmental BMD analysis (Fig 2B).

BMD assessment was initiated (proximally at the subchondral bone) and terminated (distally at the anterior tibial cortex) where at least $50 \%$ of the ring RoI was within bone per axial slice. All non-bone 

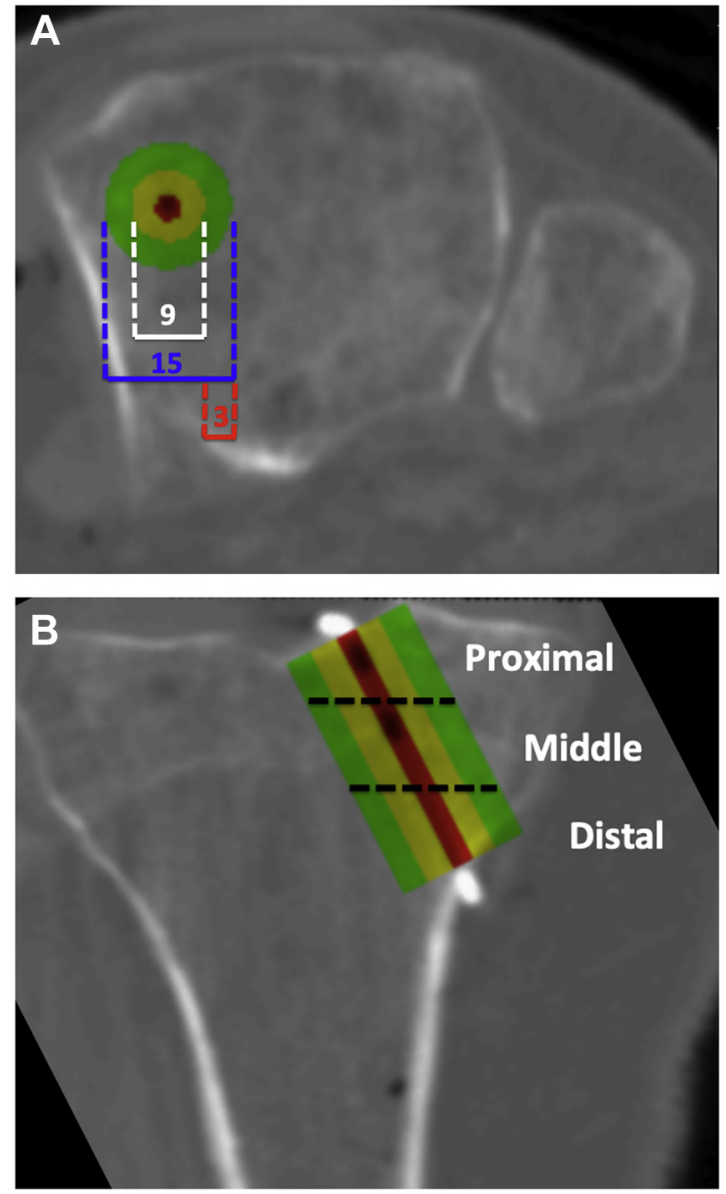

Fig 2. Cylindrical, volumetric $B M D$ of the peripheral tibial tunnel (in vitro). (A) A 3-mm ring RoI (green shade), between a $9 \mathrm{~mm}$ (yellow shade) and $15 \mathrm{~mm}$ (green, yellow, and maroon shade) circular RoI, centered over the guide pin tract (maroon shade). (B) Segmental BMD within the proximal, middle, and distal one thirds of the tibial tunnel (black dotted lines separating segments). (BMD, bone mineral density; RoI, region of interest.)

objects (radio-opaque markers and regions outside of the bone) were masked off. The average Hounsfield unit value within the RoI per axial slice was measured, and the volumetric BMD $\left(\mathrm{mg} / \mathrm{cm}^{3}\right)$ was calculated using a calibration curve derived from the phantom per manufacturer protocol.

\section{Control and ACL Injured Patient Tibial Tunnel BMD Assessment Technique}

Quantitative CT scans were acquired with the identical protocol used for the cadaveric specimens. The calibration phantom of the healthy controls and the injured knee (ACL injury cohort) were scanned within the same field of view, starting at $6 \mathrm{~cm}$ proximal to the femoral notch and ending at $10 \mathrm{~cm}$ distal to the tibial articular surface. The location of the tibial tunnel was identified and marked with custom software that allowed for manipulation of a 3-D reconstruction of the proximal tibia. The long axis of the tibial tunnel was established at approximately $55^{\circ}$ in the sagittal plane and at $65^{\circ}$ to the medial tibial plateau subchondral bone (Fig 3).

The trajectory of the tibial tunnel was directed toward the ACL footprint. The intra-articular starting point (ACL footprint) for the tibial tunnel was identified both in the sagittal and coronal plane. In the sagittal plane, the anterior to posterior distance (millimeter) of the subchondral bone at the medial tibial plateau was noted on the axial images. This value was multiplied by 0.43 ( $43 \%$ of the anterior to posterior distance) to result in the distance from the anterior subchondral bone to the center of the tibial tunnel at the articular surface, through which a horizontal line was marked. ${ }^{38,39}$ In the coronal plane, the midpoint between the medial and lateral tibial spines marked the center of the tibial tunnel at the articular surface, through which a vertical line was marked. The central axis of the tibial tunnel was positioned at the intersection of these lines (Fig 4).

Similar to the cadaveric technique, 3-mm thick ring RoIs were generated. The rings were stacked in line with the trajectory of the previously identified trajectory of a standard tibial tunnel to create a cylindrical RoI at the periphery of a potential 9-mm diameter tibial tunnel. Likewise, the cylindrical RoI was divided into 3 equal regions (proximal one-third, middle one-third, and distal one-third) for segmental BMD analysis. BMD assessment was initiated (proximally at the subchondral bone) and terminated (distally at the anterior tibial cortex) where at least $50 \%$ of the ring RoI was within bone per axial slice. All non-bone objects (region outside of the bone) were masked off. The mean

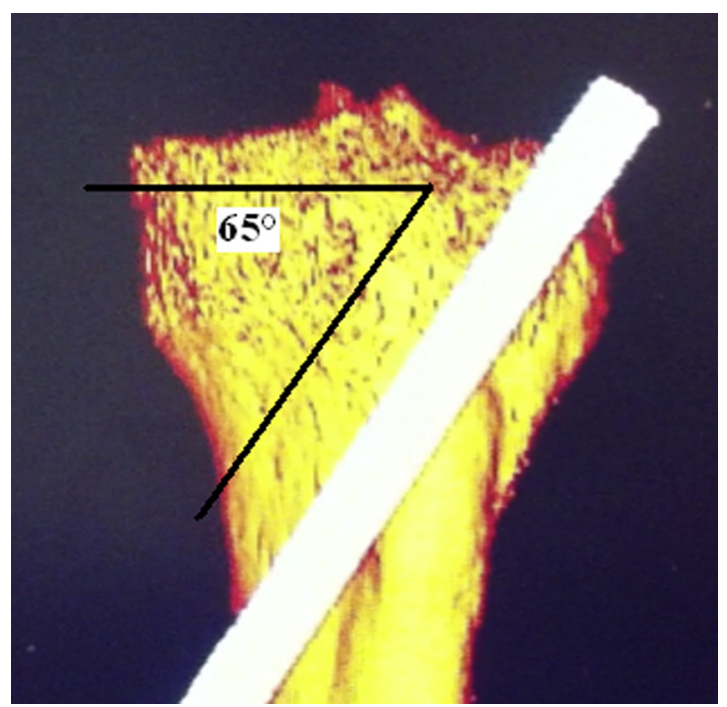

Fig 3. In vivo tibial tunnel trajectory identification. The trajectory of the tibial tunnel, $55^{\circ}$ from the articular surface in the sagittal plane and $65^{\circ}$ from the medial tibial plateau in the coronal plane is selected with the use of custom software that allows for manipulation of a 3-dimensional reconstruction of the proximal tibia. 


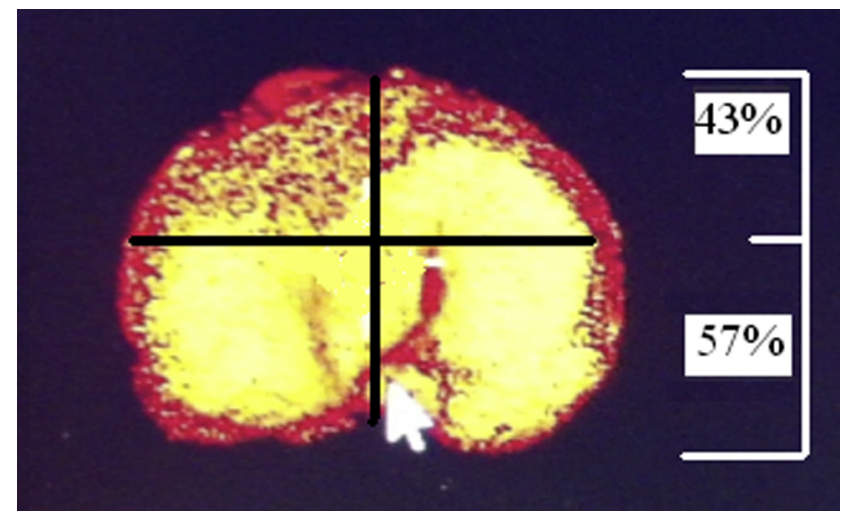

Fig 4. Identification of the ACL footprint for in vivo application. The ACL footprint is identified at the intersection (white circle) of a line draw horizontally at a distance (43\% of the anterior to posterior distance of the medial tibial plateau subchondral bone) posterior to the anterior tibial cortex and vertically at the midpoint between the medial and lateral tibial spine. (ACL, anterior cruciate ligament.)

Hounsfield unit per axial slice within the ring RoI was converted to volumetric BMD $\left(\mathrm{mg} / \mathrm{cm}^{3}\right)$ based upon the calibration phantom.

\section{Statistical Analysis}

All analyses were performed with Excel (version 14.0, Microsoft, Redmond, WA) and JMP Pro (version 14.1.0; SAS Institute Inc., Cary, NC), with sample size considered for all calculations. General statistics (mean, standard deviation, median, range, and frequencies) were performed on the subject's demographic, radiographic, and clinical information when applicable. The Wilcoxon rank-sum test was used to compare means of continuous variables, and the Fisher exact and $\chi^{2}$ tests were used to compare nominal variables, when appropriate. Spearman's rank correlation coefficient was used to test for significant correlation between demographic and radiologic information and outcome scores. $P$ values $<.05$ were considered statistically significant.

\section{Results}

A total of 90 lower extremities were analyzed, including 10 cadaveric specimens, 10 healthy controls, and 25 ACL-injured patients. Demographic and functional variables are shown in Table 1 . The healthy cohort was significantly older than their ACL injured counterparts $(35.1 \pm 10.2$ vs $25.9 \pm 7.7 ; P=.006)$ with a lower body mass index $\left(24.3 \pm 3.1 \mathrm{~kg} / \mathrm{m}^{2}\right.$ vs $27.3 \pm 1.1 \mathrm{~kg} / \mathrm{m}^{2}$; $P=.045)$. Similarly, both Lysholm $(83.3 \pm 3.9$ vs $46.8 \pm$ $23.4 ; P<.001)$ and IKDC $(96.0 \pm 7.1$ vs $50.3 \pm 17.0 ; P<$ $.001)$ knee function scores were significantly greater in the healthy cohort compared to the ACL injured cohort.

\section{Cadaveric Specimens}

The mean BMD throughout the entire length of the tibial tunnel and mean segmental BMD for cadaveric knees are listed in Table 2 and Figure 5. The mean segmental BMD was significantly different in pair-wise comparison of proximal to middle $(180.2 \pm 41.0 \mathrm{mg} /$ $\mathrm{cm}^{3}$ vs $\left.99.3 \pm 21.2 \mathrm{mg} / \mathrm{cm}^{3} ; P<.001\right)$, proximal to distal segments $\left(180.2 \pm 41.0 \mathrm{mg} / \mathrm{cm}^{3}\right.$ vs $217.4 \pm 53.1$ $\left.\mathrm{mg} / \mathrm{cm}^{3} ; P<.001\right)$, and distal to middle segments $\left(217.4 \pm 53.1 \mathrm{mg} / \mathrm{cm}^{3}\right.$ vs $99.3 \pm 21.2 \mathrm{mg} / \mathrm{cm}^{3} ; P<$ $.001)$. The comparisons between the right and left sides were not statistically significant for the total $(P=.501)$, proximal $(P=.342)$, middle $(P=.174)$, or distal segments $(P=.936)$.

\section{Healthy Subjects Study Group}

The mean BMD throughout the entire tibial tunnel and mean segmental BMD for healthy controls are shown in Table 2 and Figure 5. The mean segmental BMD was significantly different in pair-wise comparison of proximal-to-middle $(252.2 \pm 35.0$ vs $132.6 \pm$ 22.4; $P<.001)$, proximal-to-distal $(252.2 \pm 35.0$ vs $382.2 \pm 53.9 ; P<.001)$, and distal-to-middle segments $(382.2 \pm 53.9$ vs $132.6 \pm 22.4 ; P<.001)$. Mean segmental BMD was not statistically different when we compared right and left sides for total $(P=.556)$, proximal $(P=.223)$, middle $(P=.348)$, or distal segments $(P=.297)$.

\section{ACL-Injured Patients Study Group}

Of the 25 ACL-injured patients, $60 \%(n=15)$ occurred in their dominant extremity, $88 \%(\mathrm{n}=22)$ during a sporting event, and $56 \%(\mathrm{n}=15)$ due to a contact mechanism of injury. Weight-bearing was restricted for a mean of 13.2 days (range 0-42) after the injury, crutches were used for a mean of 14.8 days (range 0-42), and a knee immobilizer was used for a mean of 10.8 days (range 0-70). These patients underwent qCT scanning at a mean of 8 weeks from injury (range 1-36 weeks). The mean BMD throughout the entire length of the tibial tunnel and mean segmental BMD for the injured knee and contralateral uninjured knee in the ACL group are listed in Table 2 and Figure 5. Comparisons of mean total segment BMD revealed no differences across age, gender, and

Table 1. Baseline Demographic and Descriptive Data of Healthy and ACL-Injured Cohorts

\begin{tabular}{lccc}
\hline & $\begin{array}{c}\text { Healthy Control } \\
(\mathrm{n}=10)\end{array}$ & $\begin{array}{c}\text { ACL-Injured } \\
(\mathrm{n}=25)\end{array}$ & $P$ Value \\
\hline Age, y $\pm \mathrm{SD}$ & $35.1 \pm 10.2$ & $25.9 \pm 7.7$ & .006 \\
Sex & & & .723 \\
$\quad$ Male & $7(70 \%)$ & $20(80 \%)$ & \\
$\quad$ Female & $3(30 \%)$ & $5(20 \%)$ & .722 \\
Height, cm & $176.3 \pm 6.5$ & $175.1 \pm 9.5$ & .143 \\
Weight, kg & $75.7 \pm 12.3$ & $83.3 \pm 11.9$ & .045 \\
BMI, mean $\pm \mathrm{SD}$ & $24.3 \pm 3.1$ & $27.3 \pm 1.1$ &
\end{tabular}

NOTE. Data are n (\%) or mean \pm SD, unless stated otherwise. ACL, anterior cruciate ligament; BMI, body mass index; SD, standard deviation. 
Table 2. Comparison of Entire Tibial Tunnel and Segmental Tibial Tunnel BMD for All Cohorts

\begin{tabular}{lccrr}
\hline & & & ACL-Ruptured \\
Extremity $(\mathrm{n}=25)$ & $\begin{array}{c}\text { ACL-Uninjured } \\
\text { Extremity }(\mathrm{n}=25)\end{array}$ \\
\hline Entire tunnel & $165.8 \pm 30.5$ & Healthy $(\mathrm{n}=20)$ & $290.3 \pm 36.4$ & $300.1 \pm 35.1$ \\
Proximal one-third & $180.2 \pm 41.0$ & $255.9 \pm 28.2$ & $271.2 \pm 32.2$ & $279.1 \pm 31.3$ \\
Middle one-third & $99.3 \pm 21.2$ & $132.6 \pm 22.4$ & $167.6 \pm 31.0$ & $179.6 \pm 33.1$ \\
Distal one-third & $217.4 \pm 53.1$ & $382.2 \pm 53.9$ & $432.7 \pm 75.1$ & $441.7 \pm 74.6$ \\
Segmental differences & & & & \\
$\quad$ Proximal-middle & $81.0 \pm(63.7-98.3)$ & $119.6 \pm(104.0-135.2)$ & $103.6 \pm(88.5-118.6)$ & $100.7 \pm(84.1-117.1)$ \\
Distal-proximal & $37.1 \pm(10.1-64.2)$ & $130.0 \pm(102.0-158.0)$ & $161.5 \pm(128.8-194.3)$ & $162.6 \pm(128.3-196.9)$ \\
Distal-middle & $118.1 \pm(98.1-138.1)$ & $249.6 \pm(227.4-271.8)$ & $265.1 \pm(239.5-290.6)$ & $263 \pm(239.8-286.7)$ \\
\hline
\end{tabular}

NOTE. Values represented as mean and standard deviation reported in $\mathrm{mg} / \mathrm{cm}^{3}$, except for differences presented as the mean and the 2-tailed 95\% confidence interval.

ACL, anterior cruciate ligament; BMD, bone mineral density.

BMI for the injured $(P=.271, P=.266, P=.326)$ and uninjured extremity $(P=.209, P=.309, P=.299)$.

The mean segmental BMD in the ACL ruptured extremity was significantly different in pair-wise comparison of proximal-to-middle (271.2 \pm 32.2 vs $167.6 \pm$ 31.0; $P<.001)$, proximal-to-distal $(271.2 \pm 32.2$ vs $432.7 \pm 75.1 ; P<.001)$, and distal-to-middle segments $(432.7 \pm 75.1$ vs $167.6 \pm 31.0 ; P<.001)$. The mean segmental BMD in the contralateral ACL-uninjured extremity was also significantly different in pairwise comparison of proximal-to-middle $(279.1 \pm 31.3$ vs $179.6 \pm 33.1 ; P<.001)$, proximal-to-distal $(279.1 \pm$ 31.3 vs $441.7 \pm 74.6 ; P<.001)$, and distal-to-middle segments $(441.7 \pm 74.6$ vs $179.6 \pm 33.1 ; P<.001)$. Comparisons between the mean BMD of the ACL injured and uninjured extremity demonstrated higher values in the entire tunnel ( 290.3 vs $300.1 ; P<.001)$, proximal (271.2 vs $279.1 ; P=.002)$, middle (167.6 vs 179.6; $P<.001$ ), and distal segments (432.7 vs 441.7 ; $P=.004)$.

\section{Intergroup Analysis}

Segmental differences between the healthy, ACLinjured, and ACL-uninjured cohorts demonstrated similar changes in comparison of proximal-middle $(P=.201)$, distal-proximal $(P=.285)$, and distal-middle $(P=.619)$ (Table 2$)$. Further comparison of the healthy control and ACL injured cohorts revealed that the mean $\mathrm{BMD}$ was significantly greater in the ACL injured patients in both the injured and uninjured extremities with a difference of $34.4 \mathrm{mg} / \mathrm{cm}^{3}(P=.001)$ and $44.6 \mathrm{mg} / \mathrm{cm}^{3}(P<.001)$ in the entire tunnel, $35.0 \mathrm{mg} / \mathrm{cm}^{3}(P<.001)$ and $46.8 \mathrm{mg} / \mathrm{cm}^{3}(P<.001)$ in the middle segment, and $50.4 \mathrm{mg} / \mathrm{cm}^{3}(P=.012)$ and $59.4 \mathrm{mg} / \mathrm{cm}^{3}(P=.005)$ in the distal segment, respectively (Table 3 ). BMD was not significantly different in comparison of the healthy control and ACL injured extremity $(P=.069)$; however, the ACL-uninjured extremity had higher BMD values than the healthy cohort with a mean difference of $26.8(P=.012) \mathrm{mg} / \mathrm{cm}^{3}$.

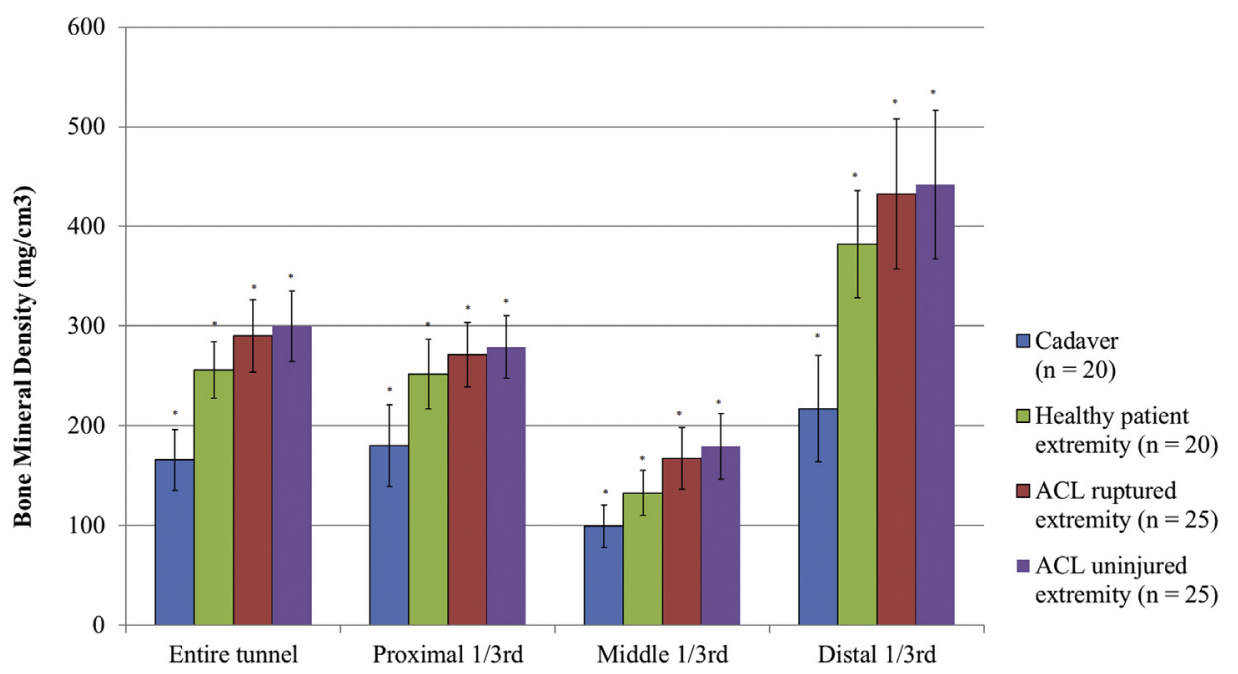

Fig 5. Mean total and segmental BMD within the region of the tibial tunnel in a cadaveric specimen, a healthy uninjured patient, and an ACL ruptured patient. Mean BMD per axial slice is much higher in the proximal (subchondral bone) and distal (anterior tibial cortex) portion of the tibial tunnel compared to the middle (cancellous bone). *Represents statistical significance of pairwise comp. (ACL, anterior cruciate ligament BMD, bone mineral density.) 
Table 3. Comparison of Entire Tibial Tunnel and Segmental Tibial Tunnel BMD Between ACL Extremities and Between ACL and Healthy Controls

\begin{tabular}{|c|c|c|c|c|}
\hline & Entire Tunnel & Proximal One-Third & Middle One-Third & Distal One-Third \\
\hline \multicolumn{5}{|l|}{$\overline{\text { ACL cohort comparison }}$} \\
\hline ACL-ruptured extremity & $290.3 \pm 36.4$ & $271.2 \pm 32.2$ & $167.6 \pm 31.0$ & $432.7 \pm 75.1$ \\
\hline ACL-uninjured extremity & $300.1 \pm 35.1$ & $279.1 \pm 31.3$ & $179.6 \pm 33.1$ & $441.7 \pm 74.6$ \\
\hline$P$ value & $<.001$ & .002 & $<.001$ & .004 \\
\hline \multicolumn{5}{|c|}{ Healthy and ACL Cohort comparison } \\
\hline Healthy cohort & $255.9 \pm 28.2$ & $252.2 \pm 35.0$ & $132.6 \pm 22.4$ & $382.2 \pm 53.9$ \\
\hline$P$ value ${ }^{*}$ & .001 & .069 & $<.001$ & .012 \\
\hline ACL-uninjured extremity & $300.1 \pm 35.1$ & $279.1 \pm 31.3$ & $179.6 \pm 33.1$ & $441.7 \pm 74.6$ \\
\hline Difference $^{\dagger}$ & $44.6 \pm(24.9-61.9)$ & $26.8 \pm(6.6-47.1)$ & $46.8 \pm(30.2-62.3)$ & $59.4 \pm(20.7-98.1)$ \\
\hline$P$ value ${ }^{\dagger}$ & $<.001$ & .012 & $<.001$ & .005 \\
\hline
\end{tabular}

The values are presented as the mean and the standard deviation reported in $\mathrm{mg} / \mathrm{cm}^{3}$, except for differences presented as the mean and the 2tailed $95 \%$ confidence interval.

$\mathrm{ACL}$, anterior cruciate ligament; $\mathrm{BMD}$, bone mineral density.

*Differences and $P$ values for the ACL-injured extremity-the healthy cohort.

${ }^{\dagger}$ The ACL-uninjured extremity—the healthy cohort.

\section{Discussion}

The main finding of this study is the development of a standardized in vitro and in vivo method to measure the volumetric BMD within the region of a standard tibial tunnel for ACL reconstruction. This technique can assess the clinical region of interest at the periphery of the tibial tunnel at the graft-bone or screw-bone interface. This method of tibial tunnel BMD assessment provided normative BMD for cadaveric specimens and was also validated in a healthy uninjured population. Most importantly, analysis between the ACLinjured and contralateral -uninjured extremity demonstrated that osteopenia can be detected within 8 weeks of injury.

Accurate assessment of the entire tibial tunnel BMD with qCT after ACL reconstruction remains an ongoing area of research. Previous investigations on qCT BMD analysis mainly focused on predicting tibial interference screw fixation strength or evaluating effects of graft preconditioning on graft tension. ${ }^{24-27}$ Weiler et al. ${ }^{40}$ did use qCT to identify a location with adequate BMD (800 $\mathrm{mg} / \mathrm{cm}^{3}$ ) within the bovine tibia to conduct fixation strength testing. Unfortunately, there were no further descriptions of the technique used for BMD assessment in that study, nor in a variety of subsequent biomechanical investigations of ACL graft fixation strength from the same authors, limiting the reproducibility of this method by others. ${ }^{41,42}$

Similarly, limited standardized methods currently exists for tibial tunnel BMD assessment with $\mathrm{qCT}$ in vivo for clinical investigations of ACL reconstructions. Muren et al. ${ }^{29}$ performed one of the first in vivo studies with qCT demonstrating no sign of increased BMD in the tibial tunnel 1 year after a bone-patellar tendon-bone graft. As a result, subsequent investigations utilized
qCT to evaluate various regions in the proximal tibia to find the optimal location for fixation into the proximal tibia based on BMD. Mariani et al. ${ }^{28}$ used qCT to evaluate the difference in trabecular bone structure between the anterior and posterior aspect of the tibia. Subsequently, Lee et al. ${ }^{43}$ determined that the anteromedial area of the proximal tibia had the highest density and was likely the most likely to accept an interference screw.

However, complete tunnel evaluation is lacking due to the imprecision of these contemporary methods and the wide variability of BMD within the proximal tibia in the axial, coronal, and sagittal planes. KhodadadyanKlostermann et al. ${ }^{37}$ noted that BMD in 40 cadaveric proximal tibias decreased significantly when traveling from proximal to metaphyseal bone and was consistently lowest in the central and anteromedial regions of the proximal tibia compared to other regions in the same axial slice. Similarly, Klein et al. ${ }^{16}$ observed significantly greater BMD, with a technique involving immersion of harvested cancellous bone cores, proximally when compared with distally within the proximal tibia of cadaveric specimens. Mariani et al. ${ }^{28}$ then reported one of the early clinical studies, demonstrating a significantly greater BMD in the anterior half of the proximal tibia as compared with the posterior half in a group of healthy young patients.

A valid assessment of BMD within the region of clinical interest for ACL reconstruction necessitates the measurement of BMD throughout the entire path of the tibial tunnel. Dunkin et al. ${ }^{44}$ used a high-resolution qCT to evaluate the bone volume fraction (bone volume/total volume) of a 2-mm thick cylinder around the periphery of the entire tibial tunnel by stacking $2 \mathrm{~mm}$ thick ring RoIs (per reconstruction slice) centered over 
the central axis of a previously extraction drilled tibial tunnel in 20 porcine tibias. Although this technique provides a valid and accurate assessment of bone quality about the tibial tunnel, BMD was not measured. The current study used qCT and a modification of the stacked ring RoI technique to provide an accurate assessment of bone quality throughout the entire path of the tibial tunnel.

The tibial tunnel BMD observed in the present study for cadaveric specimens and healthy volunteers is comparable to that reported in the literature for the proximal tibia. In 3 cohorts of cadaveric proximal tibias, with a combined mean age of 40 years (range 17-54), Nurmi et al. ${ }^{27}$ noted a mean trabecular BMD of $180 \pm$ $30 \mathrm{mg} / \mathrm{cm}^{3}, 182 \pm 43 \mathrm{mg} / \mathrm{cm}^{3}$, and $176 \pm 27 \mathrm{mg} / \mathrm{cm}^{3}$. The region evaluated by Nurmi et al. ${ }^{27}$ ( $2 \mathrm{~cm}$ below the articular surface) corresponds to the proximal segment analyzed in the current study with a similar mean segmental BMD of $180 \pm 41 \mathrm{mg} / \mathrm{cm}^{3}$ in cadaveric specimens (mean age of 76 years). In healthy volunteers, with a mean age of 39 years (range: 24 to 6 years), Sievanen et al..$^{22}$ reported a mean BMD of $167 \pm$ $33 \mathrm{mg} / \mathrm{cm}^{3}$ (range 104-235) and $349 \pm 56 \mathrm{mg} / \mathrm{cm}^{3}$ (range 263-500) in the proximal tibial for trabecular and cortical bone, respectively. The region evaluated by Sievanen et al. ${ }^{22}$ (at a distal distance of $5 \%$ of the total length of the tibia from the lateral tibial plateau subchondral bone) corresponds to the middle (trabecular) and distal (corticocancellous) segments analyzed in the current study, with similar mean middle and distal segmental BMD of $132.6 \pm 22.4 \mathrm{mg} / \mathrm{cm}^{3}$ and $382.2 \pm$ $53.9 \mathrm{mg} / \mathrm{cm}^{3}$ in healthy volunteers (mean age of 35 years), respectively.

As previously mentioned, literature defining the $\mathrm{BMD}$ within the region of the entire tibial tunnel in an ACL injured population remains limited. Bayar et al. ${ }^{2}$ noted significant BMD loss (with DEXA) about the injured knee (greatest loss at the medial proximal tibia) in 32 patients at a mean time of 24 months after an ACL disruption. Leppala et al. ${ }^{4}$ detected significant BMD loss (with DEXA) within the injured extremity (greatest loss at the proximal tibia and the patella) in 12 patients at a mean time of 12 months after sustaining a complete ACL rupture. Despite innumerable tibial fixation strength biomechanical ACL reconstruction studies, which evaluate the proximal tibia (cadaveric, porcine, or bovine) for adequate and clinically relevant bone quality, the actual BMD within the region of the tibial tunnel for an ACL-ruptured population is unknown. The current study provides normative data for BMD throughout the entire tibial tunnel in an ACL injured population.

Most importantly, a greater tibial tunnel BMD was observed in all segments of the contralateral uninjured extremity of the ACL group when compared to the ACLruptured extremity. This is in accordance with the results of previous literature which has demonstrated that ACL ruptures can result in substantial BMD loss about the knee despite ACL reconstruction. ${ }^{1-4}$ In addition, the proximal tibia is often the area of greatest bone loss..$^{2-4,8}$ While the exact pathophysiology remains unknown, attributable factors include catabolic hormones from the inciting injury, subsequent surgery, immobilization, and altered weight-bearing through the injured extremity..$^{4-7,45}$ By design, the current investigation analyzed BMD before surgical intervention and was still able to detect BMD changes between injured and uninjured extremities at a mean time of 8 weeks from the initial injury to qCT scan. Although this duration may appear relatively short, Nardo et al. ${ }^{46}$ demonstrated similar timelines with bone marrow changes being evident on MRI as soon as 10 weeks following brief immobilization. In addition, van Meer et al. ${ }^{45}$ observed a difference in DEXA measured BMD between injured and contralateral knees as soon as 10.4 weeks after an ACL injury. As such it is noteworthy the current qCT method can detect early BMD changes after an ACL injury, which may be due to trauma-related factors and/or reduced weight bearing and immobilization.

\section{Limitations}

There are several limitations to this study. First, there is potential for variability despite an attempt to standardize the technique with a single surgeon performing all the procedures and a single postimaging analysis technician performing all the processing. The authors aimed to reduce this with a test-retest demonstrating acceptable intraobserver reliability. Second, the authors used a commercially available qCT software with a stacked "ring" technique centered along a guide pin tract on axial images to create a reproducible method of tibial tunnel BMD assessment on any in-vitro specimen. However, the in vivo BMD analysis required custom software development to allow the user to define the trajectory of a standard ACL reconstruction tibial tunnel upon which the stacked "ring" technique could be aligned. ${ }^{44}$ Third, this investigation used a total of 90 specimens for analysis based on resource availability. This may represent a small sample size, but the current cohort represents comparable numbers to published cohorts and is unique in the use of qCT. In addition, the investigators attempted to use the combination of cadaveric, healthy host, and ACL ruptured patients to provide an appropriate range of BMD types.

\section{Conclusions}

A standardized method to quantitatively measure the volumetric BMD within the region of a standard tibial tunnel for ACL reconstruction was successfully developed and validated. Significant osteopenia of the injured knee occurs following ACL injury when compared to the contralateral uninjured knee. This observation has 


\section{potential clinical implications for ACL graft tibial fixation and healing.}

\section{References}

1. Takata S, Abbaspour A, Kashihara M, Nakao S, Yasui N. Unilateral chronic insufficiency of anterior cruciate ligament decreases bone mineral content and lean mass of the injured lower extremity. J Med Invest 2007;54: 316-321.

2. Bayar A, Sarikaya S, Keser S, Ozdolap S, Tuncay I, Ege A. Regional bone density changes in anterior cruciate ligament deficient knees: A DEXA study. Knee 2008;15: 373-377.

3. Zerahn B, Munk AO, Helweg J, Hovgaard C. Bone mineral density in the proximal tibia and calcaneus before and after arthroscopic reconstruction of the anterior cruciate ligament. Arthroscopy 2006;22:265-269.

4. Leppala J, Kannus P, Natri A, et al. Effect of anterior cruciate ligament injury of the knee on bone mineral density of the spine and affected lower extremity: A prospective one-year follow-Up study. Calcif Tissue Int 1999;64:357-363.

5. Jarvinen M, Kannus P. Injury of an extremity as a risk factor for the development of osteoporosis. J Bone Joint Surg Am 1997;79:263-276.

6. Sievanen H, Kannus P, Heinonen A, Oja P, Vuori I. Bone mineral density and muscle strength of lower extremities after long-term strength training, subsequent knee ligament injury and rehabilitation: A unique 2-year followup of a 26-year-old female student. Bone 1994;15:85-90.

7. Kannus P, Jarvinen M, Sievanen H, Jarvinen TA, Oja P, Vuori I. Reduced bone mineral density in men with a previous femur fracture. J Bone Miner Res 1994;9: 1729-1736.

8. Kannus P, Sievanen H, Jarvinen M, Heinonen A, Oja P, Vuori I. A cruciate ligament injury produces considerable, permanent osteoporosis in the affected knee. J Bone Miner Res 1992;7:1429-1434.

9. Brand JC Jr, Pienkowski D, Steenlage E, Hamilton D, Johnson DL, Caborn DN. Interference screw fixation strength of a quadrupled hamstring tendon graft is directly related to bone mineral density and insertion torque. Am J Sports Med 2000;28:705-710.

10. Nyland J, Kocabey Y, Caborn DN. Insertion torque pullout strength relationship of soft tissue tendon graft tibia tunnel fixation with a bioabsorbable interference screw. Arthroscopy 2004;20:379-384.

11. Safran MR, Greene HS. Avoidance and management of intra-articular complications of anterior cruciate ligament reconstruction. Instr Curse Lect 2006;55:475-488.

12. Voos JE, Drakos MC, Lorich DG, Fealy S. Proximal tibia fracture after anterior cruciate ligament reconstruction using bone-patellar tendon-bone autograft: A case report. HSS J 2008;4:20-24.

13. Liu H, Liu J, Wu Y-W, Ma Y-H, Gu S-J, Rui Y-J. Changes in local bone mineral density can guide the treatment plan for patients with rupture of the anterior cruciate ligament. Ann Palliat Med 2021;10:6388-6398.

14. Brown GA, Pena F, Grontvedt T, Labadie D, Engebretsen L. Fixation strength of interference screw fixation in bovine, young human, and elderly human cadaver knees: Influence of insertion torque, tunnel-bone block gap, and interference. Knee Surg Sports Traumatol Arthrosc 1996;3: 238-244.

15. Beynnon BD, Amis AA. In vitro testing protocols for the cruciate ligaments and ligament reconstructions. Knee Surg Sports Traumatol Arthrosc 1998;6:S70-S76 (6 suppl).

16. Klein SA, Nyland J, Caborn DN, Kocabey Y, Nawab A. Comparison of volumetric bone mineral density in the tibial region of interest for ACL reconstruction. Surg Radiol Anat 2005;27:372-376.

17. Harvey AR, Thomas NP, Amis AA. The effect of screw length and position on fixation of four-stranded hamstring grafts for anterior cruciate ligament reconstruction. Knee 2003;10:97-102.

18. Caborn DN, Nyland J, Selby J, Tetik O. Biomechanical testing of hamstring graft tibial tunnel fixation with bioabsorbable interference screws. Arthroscopy 2003;19:991-996.

19. Chang HC, Nyland J, Nawab A, Burden R, Caborn DN. Biomechanical comparison of the bioabsorbable RetroScrew system, BioScrew XtraLok with stress equalization tensioner, and 35-mm Delta Screws for tibialis anterior graft-tibial tunnel fixation in porcine tibiae. Am J Sports Med 2005;33:1057-1064.

20. Yoo JC, Ahn JH, Kim JH, et al. Biomechanical testing of hybrid hamstring graft tibial fixation in anterior cruciate ligament reconstruction. Knee 2006;13:455-459.

21. Boyd SK, Matyas JR, Wohl GR, Kantzas A, Zernicke RF. Early regional adaptation of periarticular bone mineral density after anterior cruciate ligament injury. J Appl Physiol 2000;89:2359-2364.

22. Sievanen H, Koskue V, Rauhio A, Kannus P, Heinonen A, Vuori I. Peripheral quantitative computed tomography in human long bones: evaluation of in vitro and in vivo precision. J Bone Miner Res 1998;13:871-882.

23. Adolphson P, von Sivers K, Dalen N, Jonsson U, Dahlborn M. Bone and muscle mass after hip arthroplasty. A quantitative computed tomography study in 20 arthrosis cases. Acta Orthop Scand 1993;64:181-184.

24. Jarvinen TL, Nurmi JT, Sievanen H. Bone density and insertion torque as predictors of anterior cruciate ligament graft fixation strength. Am J Sports Med 2004;32:1421-1429.

25. Nurmi JT, Kannus P, Sievanen H, Jarvinen M, Jarvinen TL. Compaction drilling does not increase the initial fixation strength of the hamstring tendon graft in anterior cruciate ligament reconstruction in a cadaver model. Am J Sports Med 2003;31:353-358.

26. Nurmi JT, Jarvinen TL, Kannus P, Sievanen H, Toukosalo J, Jarvinen M. Compaction versus extraction drilling for fixation of the hamstring tendon graft in anterior cruciate ligament reconstruction. Am J Sports Med 2002;30:167-173.

27. Nurmi JT, Kannus P, Sievanen H, Jarvela T, Jarvinen M, Jarvinen TL. Interference screw fixation of soft tissue grafts in anterior cruciate ligament reconstruction: Part 2: effect of preconditioning on graft tension during and after screw insertion. Am J Sports Med 2004;32:418-424.

28. Mariani PP, Margheritini F, Bellelli A. Bone mineral density of the proximal metaphysis of tibia: Clinical relevance in posterior cruciate ligament reconstruction. Knee Surg Sports Traumatol Arthrosc 2005;13:263-267. 
29. Muren O, Brosjo E, Dahlstedt L, Dahlborn M, Dalen N. No bone ingrowth into the tibia tunnel in anterior cruciate ligament-reconstructed patients: A l-year prospective quantified CT study of 10 patients reconstructed with an autologous bone-patellar tendon-bone graft. Acta Orthop Scand 2001;72:481-486.

30. Lysholm J, Gillquist J. Evaluation of knee ligament surgery results with special emphasis on use of a scoring scale. Am J Sports Med 1982;10:150-154.

31. Anderson AF, Irrgang JJ, Kocher MS, Mann BJ, Harrast JJ. The International Knee Documentation Committee Subjective Knee Evaluation Form: Normative data. Am J Sports Med 2006;34:128-135.

32. Tegner Y, Lysholm J. Rating systems in the evaluation of knee ligament injuries. Clin Orthop Relat Res 1985;198: 43-49.

33. Howell SM, Gittins ME, Gottlieb JE, Traina SM, Zoellner TM. The relationship between the angle of the tibial tunnel in the coronal plane and loss of flexion and anterior laxity after anterior cruciate ligament reconstruction. Am J Sports Med 2001;29: 567-574.

34. Rhee PC, Levy BA, Stuart MJ, Thoreson A, An KN, Dahm DL. A biomechanical comparison of the Delta screw and RetroScrew tibial fixation on initial intra-articular graft tension. Knee Surg Sports Traumatol Arthrosc $2011 ; 19: 781-786$

35. Rhee PC, Dahm DL, Stuart MJ, Thoreson A, An KN, Levy BA. Delta screw versus RetroScrew tibial fixation for ACL reconstruction. Knee Surg Sports Traumatol Arthrosc $2011 ; 19:$ S94-S100 (suppl 1).

36. Genant HK, Engelke K, Fuerst T, et al. Noninvasive assessment of bone mineral and structure: State of the art. J Bone Miner Res 1996;1 1:707-730.

37. Khodadadyan-Klostermann C, von Seebach M, Taylor WR, Duda GN, Haas NP. Distribution of bone mineral density with age and gender in the proximal tibia. Clin Biomech (Bristol, Avon) 2004;19:370-376.
38. Amis AA, Jakob RP. Anterior cruciate ligament graft positioning, tensioning and twisting. Knee surg Sports Traumatol Arthrosc 1998;6:S2-S12 (suppl 1).

39. Musahl V, Burkart A, Debski RE, Van Scyoc A, Fu FH, Woo SL. Anterior cruciate ligament tunnel placement: Comparison of insertion site anatomy with the guidelines of a computer-assisted surgical system. Arthroscopy 2003;19: 154-160.

40. Weiler A, Windhagen HJ, Raschke MJ, Laumeyer A, Hoffmann RF. Biodegradable interference screw fixation exhibits pull-out force and stiffness similar to titanium screws. Am J Sports Med 1998;26:1 19-126.

41. Weiler A, Hoffmann RF, Siepe CJ, Kolbeck SF, Sudkamp NP. The influence of screw geometry on hamstring tendon interference fit fixation. Am J Sports Med 2000;28:356-359.

42. Weiler A, Richter M, Schmidmaier G, Kandziora F, Sudkamp NP. The EndoPearl device increases fixation strength and eliminates construct slippage of hamstring tendon grafts with interference screw fixation. Arthroscopy 2001;17:353-359.

43. Lee YS, Nam SW, Hwang CH, Lee BK. Computed tomography based evaluation of the bone mineral density around the fixation area during knee ligament reconstructions: Clinical relevance in the choice of fixation method. Knee 2012;19:793-796.

44. Dunkin BS, Nyland J, Duffee AR, Brunelli JA, Burden R, Caborn D. Soft tissue tendon graft fixation in serially dilated or extraction-drilled tibial tunnels: Aa porcine model study using high-resolution quantitative computerized tomography. Am J Sports Med 2007;35:448-457.

45. van Meer BL, Waarsing JH, van Eijsden WA, et al. Bone mineral density changes in the knee following anterior cruciate ligament rupture. Osteoarthritis Cartilage 2014;22: 154-161.

46. Nardo L, Sandman DN, Virayavanich W, et al. Bone marrow changes related to disuse. Eur Radiol 2013;23: 3422-3431. 